\title{
COMMENT \\ COVID-19 impact on research, lessons learned from COVID-19 research, implications for pediatric research
}

\author{
Debra L. Weiner ${ }^{1,2}$, Vivek Balasubramaniam ${ }^{3}$, Shetal I. Shah ${ }^{4}$ and Joyce R. Javier ${ }^{5,6}$ on behalf of the Pediatric Policy Council \\ Pediatric Research (2020) 88:148-150; https://doi.org/10.1038/s41390-020-1006-3
}

The COVID-19 pandemic has resulted in unprecedented research worldwide. The impact on research in progress at the time of the pandemic, the importance and challenges of real-time pandemic research, and the importance of a pediatrician-scientist workforce are all highlighted by this epic pandemic. As we navigate through and beyond this pandemic, which will have a long-lasting impact on our world, including research and the biomedical research enterprise, it is important to recognize and address opportunities and strategies for, and challenges of research and strengthening the pediatrician-scientist workforce.

The first cases of what is now recognized as SARS-CoV-2 infection, termed COVID-19, were reported in Wuhan, China in December 2019 as cases of fatal pneumonia. By February 26, 2020, COVID-19 had been reported on all continents except Antarctica. As of May 4, 2020, 3.53 million cases and 248,169 deaths have been reported from 210 countries. $^{1}$

\section{IMPACT OF COVID-19 ON ONGOING RESEARCH}

The impact on research in progress prior to COVID-19 was rapid, dramatic, and no doubt will be long term. The pandemic curtailed most academic, industry, and government basic science and clinical research, or redirected research to COVID19. Most clinical trials, except those testing life-saving therapies, have been paused, and most continuing trials are now closed to new enrollment. Ongoing clinical trials have been modified to enable home administration of treatment and virtual monitoring to minimize participant risk of COVID-19 infection, and to avoid diverting healthcare resources from pandemic response. In addition to short- and long-term patient impact, these research disruptions threaten the careers of physician-scientists, many of whom have had to shift efforts from research to patient care. To protect research in progress, as well as physicianscientist careers and the research workforce, ongoing support is critical. NIH (https://grants.nih.gov/policy/natural-disasters/ corona-virus.htm), PCORI (https://www.pcori.org/fundingopportunities/applicant-and-awardee-faqs-related-covid-19), and other funders acted swiftly to provide guidance on proposal submission and award management, and implement allowances that enable grant personnel to be paid and time lines to be relaxed. Research institutions have also implemented strategies to mitigate the long-term impact of research disruptions. Support throughout and beyond the pandemic to retain currently well-trained research personnel and research support teams, and to accommodate loss of research assets, including laboratory supplies and study participants, will be required to complete disrupted research and ultimately enable new research.

In the long term, it is likely that the pandemic will force reallocation of research dollars at the expense of research areas funded prior to the pandemic. It will be more important than ever for the pediatric research community to engage in discussion and decisions regarding prioritization of funding goals for dedicated pediatric research and meaningful inclusion of children in studies. The recently released 2020 National Institute of Child Health and Development (NICHD) strategic plan that engaged stakeholders, including scientists and patients, to shape the goals of the Institute, will require modification to best chart a path toward restoring normalcy within pediatric science.

\section{COVID-19 RESEARCH}

This global pandemic once again highlights the importance of research, stable research infrastructure, and funding for public health emergency (PHE)/disaster preparedness, response, and resiliency. The stakes in this worldwide pandemic have never been higher as lives are lost, economies falter, and life has radically changed. Ultimate COVID-19 mitigation and crisis resolution is dependent on high-quality research aligned with top priority societal goals that yields trustworthy data and actionable information. While the highest priority goals are treatment and prevention, biomedical research also provides data critical to manage and restore economic and social welfare.

Scientific and technological knowledge and resources have never been greater and have been leveraged globally to perform COVID-19 research at warp speed. The number of studies related to COVID-19 increases daily, the scope and magnitude of engagement is stunning, and the extent of global collaboration unprecedented. On January 5, 2020, just weeks after the first cases of illness were reported, the genetic sequence, which identified the pathogen as a novel coronavirus, SARS-CoV-2, was released, providing information essential for identifying and developing treatments, vaccines, and diagnostics. As of May 3, 20201133 COVID-19 studies, including 148 related to hydroxychloroquine, 13

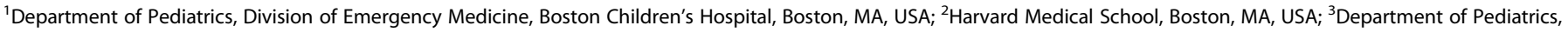

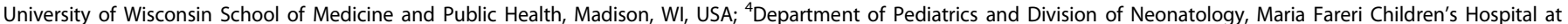

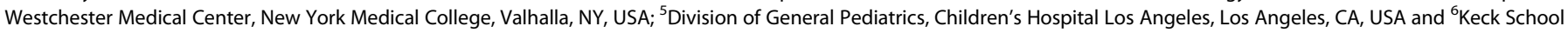
of Medicine, University of Southern California, Los Angeles, CA, USA

Correspondence: Debra L. Weiner (debra.weiner@childrens.harvard.edu)

Members of the Pediatric Policy Council are listed below Author contributions.

Received: 7 May 2020 Accepted: 21 May 2020

Published online: 16 June 2020 
to remdesivir, 50 to vaccines, and 100 to diagnostic testing, were registered on ClinicalTrials.gov, and 980 different studies on the World Health Organization's International Clinical Trials Registry Platform (WHO ICTRP), made possible, at least in part, by use of data libraries to inform development of antivirals, immunomodulators, antibody-based biologics, and vaccines. On April 7, 2020, the FDA launched the Coronavirus Treatment Acceleration Program (CTAP) (https://www.fda.gov/drugs/coronavirus-covid19-drugs/coronavirus-treatment-acceleration-program-ctap). On April 17, 2020, NIH announced a partnership with industry to expedite vaccine development (https://www.nih.gov/newsevents/news-releases/nih-launch-public-private-partnershipspeed-covid-19-vaccine-treatment-options). As of May 1, 2020, remdesivir (Gilead), granted FDA emergency use authorization, is the only approved therapeutic for COVID-19. ${ }^{2}$

The pandemic has intensified research challenges. In a rush for data already thousands of manuscripts, news reports, and blogs have been published, but to date, there is limited scientifically robust data. Some studies do not meet published clinical trial standards, which now include FDA's COVID-19-specific standards, ${ }^{3-5}$ and/or are published without peer review. Misinformation from studies diverts resources from development and testing of more promising therapeutic candidates and has endangered lives. Ibuprofen, initially reported as unsafe for patients with COVID-19, resulted in a shortage of acetaminophen, endangering individuals for whom ibuprofen is contraindicated. Hydroxychloroquine initially reported as potentially effective for treatment of COVID-19 resulted in shortages for patients with autoimmune diseases. Remdesivir, in rigorous trials, showed decrease in duration of COVID-19, with greater effect given early. ${ }^{6}$ Given the limited availability and safety data, the use outside clinical trials is currently approved only for severe disease. Vaccines typically take 10-15 years to develop. As of May 3, 2020, of nearly 100 vaccines in development, 8 are in trial. Several vaccines are projected to have emergency approval within 12-18 months, possibly as early as the end of the year, ${ }^{7}$ still an eternity for this pandemic, yet too soon for long-term effectiveness and safety data. Antibody testing, necessary for diagnosis, therapeutics, and vaccine testing, has presented some of the greatest research challenges, including validation, timing, availability and prioritization of testing, interpretation of test results, and appropriate patient and societal actions based on results. ${ }^{8}$ Relaxing physical distancing without data regarding test validity, duration, and strength of immunity to different strains of COVID-19 could have catastrophic results. Understanding population differences and disparities, which have been further exposed during this pandemic, is critical for response and long-term pandemic recovery. The "Equitable Data Collection and Disclosure on COVID-19 Act" calls for the CDC (Centers for Disease Control and Prevention) and other HHS (United States Department of Health \& Human Services) agencies to publicly release racial and demographic information (https:// bass.house.gov/sites/bass.house.gov/files/Equitable\%20Data\% 20Collection\%20and\%20Dislosure\%20on\%20COVID19\% 20Act_FINAL.pdf)

Trusted sources of up-to-date, easily accessible information must be identified (e.g., WHO https://www.who.int/emergencies/ diseases/novel-coronavirus-2019/global-research-on-novel-

coronavirus-2019-ncov, CDC https://www.cdc.gov/coronavirus/ 2019-nCoV/hcp/index.html, and for children AAP (American Academy of Pediatrics) https://www.aappublications.org/cc/ covid-19) and should comment on quality of data and provide strategies and crisis standards to guide clinical practice.

Long-term, lessons learned from research during this pandemic could benefit the research enterprise worldwide beyond the pandemic and during other PHE/disasters with strategies for balancing multiple novel approaches and high-quality, timeefficient, cost-effective research. This challenge, at least in part, can be met by appropriate study design, collaboration, patient registries, automated data collection, artificial intelligence, data sharing, and ongoing consideration of appropriate regulatory approval processes. In addition, research to develop and evaluate innovative strategies and technologies to improve access to care, management of health and disease, and quality, safety, and cost effectiveness of care could revolutionize healthcare and healthcare systems. During PHE/disasters, crisis standards for research should be considered along with ongoing and just-in-time PHE/ disaster training for researchers willing to share information that could be leveraged at time of crisis. A dedicated funded core workforce of $\mathrm{PHE} /$ disaster researchers and funded infrastructure should be considered, potentially as a consortium of networks, that includes physician-scientists, basic scientists, social scientists, mental health providers, global health experts, epidemiologists, public health experts, engineers, information technology experts, economists and educators to strategize, consult, review, monitor, interpret studies, guide appropriate clinical use of data, and inform decisions regarding effective use of resources for PHE/ disaster research.

\section{DIFFERENCES BETWEEN ADULT AND PEDIATRIC COVID-19, THE NEED FOR PEDIATRIC RESEARCH}

As reported by the CDC, from February 12 to April 2, 2020, of 149,760 cases of confirmed COVID-19 in the United States, 2572 $(1.7 \%)$ were children aged $<18$ years, similar to published rates in China. ${ }^{9}$ Severe illness has been rare. Of 749 children for whom hospitalization data is available, 147 (20\%) required hospitalization (5.7\% of total children), and 15 of 147 required ICU care $(2.0 \%$, $0.58 \%$ of total). Of the 95 children aged $<1$ year, 59 (62\%) were hospitalized, and 5 (5.3\%) required ICU admission. Among children there were three deaths. Despite children being relatively spared by COVID-19, spread of disease by children, and consequences for their health and pediatric healthcare are potentially profound with immediate and long-term impact on all of society.

We have long been aware of the importance and value of pediatric research on children, and society. COVID-19 is no exception and highlights the imperative need for a pediatricianscientist workforce. Understanding differences in epidemiology, susceptibility, manifestations, and treatment of COVID-19 in children can provide insights into this pathogen, pathogen-host interactions, pathophysiology, and host response for the entire population. Pediatric clinical registries of COVID-infected, COVIDexposed children can provide data and specimens for immediate and long-term research. Of the 1133 COVID-19 studies on ClinicalTrials.gov, 202 include children aged $\leq 17$ years. Sixty-one of the 681 interventional trials include children. With less diagnostic testing and less pediatric research, we not only endanger children, but also adults by not identifying infected children and limiting spread by children.

Pediatric considerations and challenges related to treatment and vaccine research for COVID-19 include appropriate dosing, pediatric formulation, and pediatric specific short- and long-term effectiveness and safety. Typically, initial clinical trials exclude children until safety has been established in adults. But with time of the essence, deferring pediatric research risks the health of children, particularly those with special needs. Considerations specific to pregnant women, fetuses, and neonates must also be addressed. Childhood mental health in this demographic, already struggling with a mental health pandemic prior to COVID-19, is now further challenged by social disruption, food and housing insecurity, loss of loved ones, isolation from friends and family, and exposure to an infodemic of pandemic-related information. Interestingly, at present mental health visits along with all visits to pediatric emergency departments across the United States are dramatically decreased. Understanding factors that mitigate and worsen psychiatric symptoms should be a focus of research, and ideally will result in strategies for prevention and management in 
the long term, including beyond this pandemic. Social well-being of children must also be studied. Experts note that the pandemic is a perfect storm for child maltreatment given that vulnerable families are now socially isolated, facing unemployment, and stressed, and that children are not under the watch of mandated reporters in schools, daycare, and primary care. ${ }^{10}$ Many states have observed a decrease in child abuse reports and an increase in severity of emergency department abuse cases. In the short term and long term, it will be important to study the impact of access to care, missed care, and disrupted education during COVID-19 on physical and cognitive development.

Training and supporting pediatrician-scientists, such as through $\mathrm{NIH}$ physician-scientist research training and career development programs (https://researchtraining.nih.gov/infographics/physicianscientist) at all stages of career, as well as fostering research for fellows, residents, and medical students willing to dedicate their research career to, or at least understand implications of their research for, PHE/disasters is important for having an ongoing, as well as a just-in-time surge pediatric-focused PHE/ disaster workforce. In addition to including pediatric experts in collaborations and consortiums with broader population focus, consideration should be given to pediatric-focused multi-institutional, academic, industry, and/or government consortiums with infrastructure and ongoing funding for virtual training programs, research teams, and multidisciplinary oversight.

\section{SUMMARY}

The impact of the COVID-19 pandemic on research and research in response to the pandemic once again highlights the importance of research, challenges of research particularly during PHE/ disasters, and opportunities and resources for making research more efficient and cost effective. New paradigms and models for research will hopefully emerge from this pandemic. The importance of building sustained PHE/disaster research infrastructure and a research workforce that includes training and funding for pediatrician-scientists and integrates the pediatrician research workforce into high-quality research across demographics, supports the pediatrician-scientist workforce and pipeline, and benefits society.

\section{AUTHOR CONTRIBUTIONS}

All authors made substantial contributions to conception and design, data acquisition and interpretation, drafting the manuscript, and providing critical revisions. All authors approve this final version of the manuscript.

\section{PEDIATRIC POLICY COUNCIL}

Scott C. Denne, MD, Chair, Pediatric Policy Council; Mona Patel, MD, Representative to the PPC from the Academic Pediatric Association; Jean L. Raphael, MD, MPH, Representative to the PPC from the Academic Pediatric Association; Jonathan Davis, MD, Representative to the PPC from the American Pediatric Society; DeWayne Pursley, MD, MPH, Representative to the PPC from the American Pediatric Society; Tina Cheng, MD, MPH, Representative to the PPC from the Association of Medical School Pediatric Department Chairs; Michael Artman, MD, Representative to the PPC from the Association of Medical School Pediatric Department Chairs; Shetal Shah, MD, Representative to the PPC from the Society for Pediatric Research; Joyce Javier, MD, MPH, MS, Representative to the PPC from the Society for Pediatric Research.

\section{ADDITIONAL INFORMATION}

Competing interests: The authors declare no competing interests.

Publisher's note Springer Nature remains neutral with regard to jurisdictional claims in published maps and institutional affiliations.

\section{REFERENCES}

1. Johns Hopkins Coronavirus Resource Center. Covid-19 Case Tracker. Center for Systems Science and Engineering (CSSE) at Johns Hopkins University (JHU). https://coronavirus.jhu.edu/map.html (2020).

2. US Food and Drug Administration. Coronavirus (COVID-19) update: FDA issues emergency use authorization for potential COVID-19 treatment. FDA News Release. https://www.fda.gov/news-events/press-announcements/coronavirus-covid-19update-fda-issues-emergency-use-authorization-potential-covid-19-treatment (2020).

3. Evans, S. R. Fundamentals of clinical trial design. J. Exp. Stroke Transl. Med. 3, 19-27 (2010).

4. Antman, E. M. \& Bierer, B. E. Standards for clinical research: keeping pace with the technology of the future. Circulation 133, 823-825 (2016).

5. Food and Drug Administration. FDA guidance on conduct of clinical trials of medical products during COVID-19 public health emergency. Guidance for Industry, Investigators and Institutional Review Boards. https://www.fda.gov/ regulatory-information/search-fda-guidance-documents/fda-guidance-conductclinical-trials-medical-products-during-covid-19-public-health-emergency (2020).

6. National Institutes of Health. NIH clinical trials shows remdesivir accelerates recovery from advanced COVID-19. NIH New Releases. https://www.nih.gov/newsevents/news-releases/nih-clinical-trial-shows-remdesivir-accelerates-recoveryadvanced-covid-19\#.XrIX75ZmQeQ.email (2020).

7. Radcliffe, S. Here's exactly where we are with vaccines and treatments for COVID19. Health News. https://www.healthline.com/health-news/heres-exactly-wherewere-at-with-vaccines-and-treatments-for-covid-19 (2020).

8. Abbasi, J. The promise and peril of antibody testing for COVID-19. JAMA. https:// doi.org/10.1001/jama.2020.6170 (2020).

9. CDC COVID-19 Response Team. Coronavirus disease 2019 in children-United States, February 12-April 2, 2020. Morb. Mortal Wkly Rep. 69, 422-426 (2020).

10. Agarwal, N. Opinion: the coronavirus could cause a child abuse epidemic. The New York Times. https://www.nytimes.com/2020/04/07/opinion/coronavirus-child-abuse. html (2020). 